Article

\title{
An Accurate Clinical Implication Assessment for Diabetes Mellitus Prevalence Based on a Study from Nigeria
}

\author{
Muhammad Noman Sohail ${ }^{1, * \mathbb{D}}$, Ren Jiadong ${ }^{1}$, Musa Uba Muhammad ${ }^{1}$, \\ Sohaib Tahir Chauhdary ${ }^{2}$, Jehangir Arshad ${ }^{2}(1)$ and Antony John Verghese ${ }^{3}$ \\ 1 Department of Information Sciences and Technology, Yanshan University, Qinhuangdao 066000, China; \\ jdren@ysu.edu.cn (R.J.); musaubamuhammad@stumail.ysu.edu.cn (M.U.M.) \\ 2 Department of Electrical and Computer Engineering, COMSATS University Islamabad, Islamabad 43600, \\ Pakistan; sohaibchauhdary@hotmail.com (S.T.C.); jehangir@cuisahiwal.edu.pk (J.A.) \\ 3 Department of Management, American Hotel and Lodging Association, New York, NY 10006, USA; \\ antomatter@gmail.com \\ * Correspondence: mn.sohail@stumail.ysu.edu.cn; Tel.: +86-1503-237-0085
}

Received: 9 April 2019; Accepted: 10 May 2019; Published: 15 May 2019

\begin{abstract}
The increasing rate of diabetes is found across the planet. Therefore, the diagnosis of pre-diabetes and diabetes is important in populations with extreme diabetes risk. In this study, a machine learning technique was implemented over a data mining platform by employing Rule classifiers (PART and Decision table) to measure the accuracy and logistic regression on the classification results for forecasting the prevalence in diabetes mellitus patients suffering simultaneously from other chronic disease symptoms. The real-life data was collected in Nigeria between December 2017 and February 2019 by applying ten non-intrusive and easily available clinical variables. The results disclosed that the Rule classifiers achieved a mean accuracy of $98.75 \%$. The error rate, precision, recall, F-measure, and Matthew's correlation coefficient MCC were $0.02 \%, 0.98 \%, 0.98 \%$, $0.98 \%$, and $0.97 \%$, respectively. The forecast decision, achieved by employing a set of 23 decision rules (DR), indicates that age, gender, glucose level, and body mass are fundamental reasons for diabetes, followed by work stress, diet, family diabetes history, physical exercise, and cardiovascular stroke history. The study validated that the proposed set of DR is practical for quick screening of diabetes mellitus patients at the initial stage without intrusive medical tests and was found to be effective in the initial diagnosis of diabetes.
\end{abstract}

Keywords: data mining; cluster; clinical implications; diabetes; epidemiology; forecast; PART; Decision table; Weka; real-life patients; regression; machine learning

\section{Introduction}

Diabetes mellitus (DM) is an exponentially growing disease across the developing countries of the 21st century. Diabetes mellitus has now become a worldwide challenge and identified as the risk factor of other chronic diseases such as hyperosmolar, diabetic ketoacidosis, and hyperglycemia and, in extreme cases, death. Furthermore, diabetes also causes long-term complications, for instance, cardiovascular disease, heart stroke, kidney failure, chronic ulcers, blindness, damage to the eyes, and many more [1]. Williams wrote in his book "Williams textbook of Endocrinology" [2] that around 385 million people were affected with diabetes in 2013. If Diabetes mellitus is left untreated, this figure can get higher; it can even lead to death. Around 425 million people had diabetes in the world by the survey report of the International Diabetes Federation (IDF) in 2015 [3]. Also, the report indicates that 
382 million people around the globe are affected by diabetes in developing countries alone and Africa has $4.9 \%$ from this ratio.

By the World Health Organization (WHO) [4], 321,100 deaths occurred in the African region due to diabetes, out of which $79 \%$ of the population was under the age of 60 ; this is the maximum number in any region of the world. The ratio of diabetes mellitus patients in rural and urban areas of Nigeria varies from $0.67 \%$ to $12 \%$, and this ratio has been estimated to more than double over the past two decades [5]. According to the IDF report, the ratio of undiagnosed diabetic people in sub-Saharan Africa (SSA) is estimated at $87 \%$, out of which $8.7 \%$ in the male and $8.9 \%$ in the female population of Nigeria. It is due to the lack of information and government resources [6]. In addition, the American Diabetes Association (ADA) estimates that the prevalence was estimated in Nigeria as $20.01 \%$ in both the male and female population [7]. Compared with the world population, the Nigerian health organizations pointed out that the diabetes prevalence was $4.7 \%$ in 2010 and it was projected to be $5.8 \%$ by 2030 and even exceed $10 \%$ by 2040 [8]. However, this estimate comes from rural areas, and it is expected to be more in urban slums.

In this study, the fundamental objective was to develop a quick and accurate prediction assessment scheme by using easily observable clinical features to identify patients with a high risk of diabetes. For this purpose, the machine learning Rule classifiers (projective adaptive response theory (PART) and Decision table) were used on the Weka 3.9.2 platform for acquiring accuracy in classification assumptions. Afterward, the logistic regression (LR) was utilized on the classification results to predict and forecast patients with a high risk of diabetes. This research can be applied to diabetes mellitus patients who cannot afford the expenses of the medical laboratory and specifically those in remote areas or villages with low socioeconomic status and excessive epidemiological risk.

Correspondingly, the remaining paper is structured as follows: Section 2 explains the material and methodology after the background description, Section 3 reviews the results, Section 4 discusses the results and limitations, and Section 5 concludes the findings.

\section{Background}

Numerous authors work to develop appropriate disease prediction algorithms. For instance, Lélis et al. applied seven classification techniques in a Brazilian investigation to make a diagnosis of meningococcal meningitis and verified that the model is affordable and accurate [9]. Susanne et al. proposed a mathematical model to forecast the prevalence of diabetes by using attributes of sex, age, risk factor status, and T2DM (type 2 diabetes mellitus) status and found T2DM prevalence is projected to increase by $43 \%$, and the incidence is projected to increase $147 \%$ by 2050 in Qatar [10]. Choi et al. applied support vector machine (SVM) and artificial neural network (ANN) to screen the pre-diabetes of 9251 individuals and performed a systematic assessment of the models using external and internal cross-validation and concluded that the results of the SVM method are better than the ANN [11]. Amir et al. proposed a time series prediction model for the diagnosis of diabetes patients [12]. In addition, Olivera et al. utilized machine learning algorithms from ELSA-Brazil and identified individuals with the highest risk of undiagnosed diabetes from readily available clinical data [13]. Sohail et al. performed the classification results on Weka by machine learning by utilizing the dataset of different diseases and concluded the accuracy ratio of the decision tree (86\%), the Bayesian network $(90 \%)$, the naïve Bayesian (76\%), the fuzzy cognitive map (94\%), and K-nearest neighbor (KNN) (94\%) [14]. Parampreet et al. applied a cloud-based framework with the help of sensor devices to initially screen patients for the prediction of diabetes [15]. Further, Hassan et al. proposed a unified machine-learning framework for diabetes predications in big data [16]. There is considerable interest in determining how different classification techniques from machine learning can be utilized as disease prediction tools [17-21]. These tools have been used to diagnose diabetes [22], glaucoma [23], meningitis [24], coronary artery disease [25], asthma [26], cancer [27], hypertension [28], heart arrhythmia [29], tuberculosis [30], and other diseases [31,32]. 


\section{Material and Methods}

\subsection{Ethical Consents}

The study was approved by the Natural Science Foundation of China Hebei province, the Yanshan University ethics committee, and all experiments and simulation procedures conformed to the Declaration of Helsinki. All participants provided written informed consent after having all procedures explained to them both verbally and in writing.

\subsection{Model Framework}

Figure 1 shows the assessment framework used in this study for diabetes patient screening. The assessments were performed in a total of six steps. Initially, the real-life diabetes mellitus data were acquired and preprocessed for selection of appropriate attributes. Afterward, this data was utilized for evaluation and assessment. Secondly, the updated plugins of two machine learning Rule classifiers (PART and Decision table) were used on Weka version 3.9.2 "data mining platform" for classification measurements and Rule assessment [33]. In addition, the logistic regression method was utilized on the results of the machine learning classifiers to forecast the rule assessment.

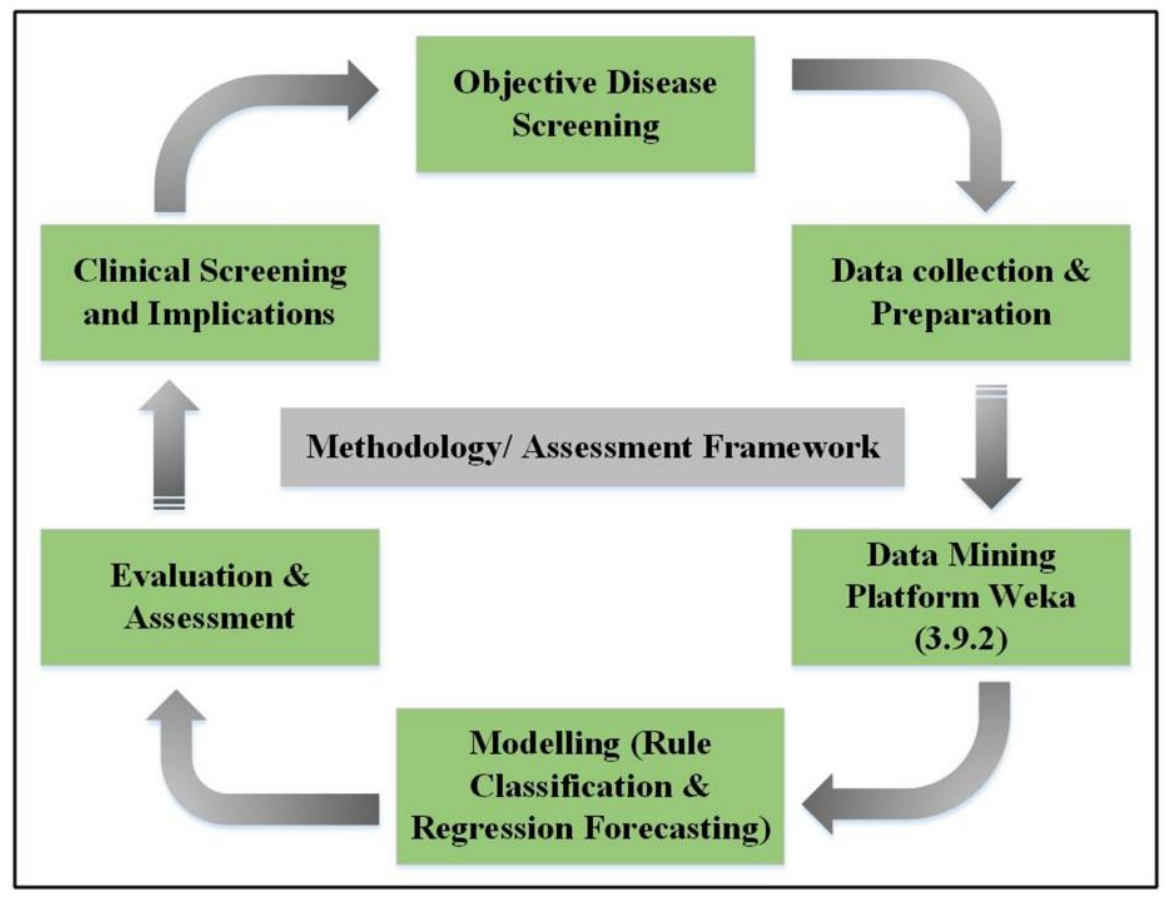

Figure 1. Assessment framework used in this study for clinical implication screening.

\subsection{Data Collection and Explanation}

The real-life diabetes mellitus data of 1257 patients from December 2017 to February 2019 were acquired from four main hospitals across Nigeria and carefully examined. Figure 2 demonstrate the collection flow of data gathered from four principal hospitals in Nigeria namely Abdullahi Wase Specialist Hospital (22.75\%), Ajingi General Hospital (22.04\%), Federal Medical Center Birnin-Kudu $(26.81 \%)$, and Gaya General Hospital (28.40\%) located in the northwestern region of Nigeria. The data were collected through questionnaires, verbal interviews, and by consultation of the medical specialist after the ethics committee of the institute where the research was carried out approved the study protocols. The data collection flow of diabetes patients from the mentioned hospitals is shown in Figure 2, and the number of patients in each hospital is shown in Figure 3. 


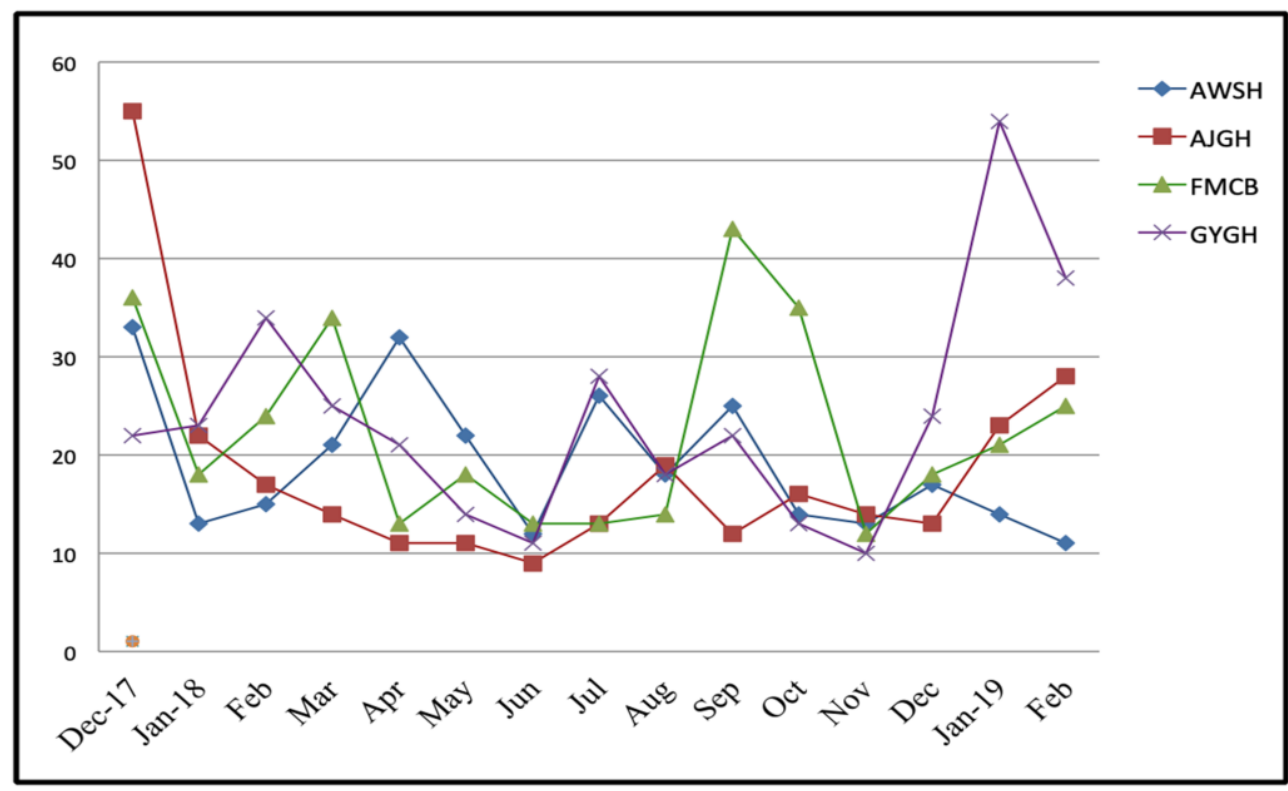

Figure 2. The data collection flow of diabetes patients from the four hospitals.

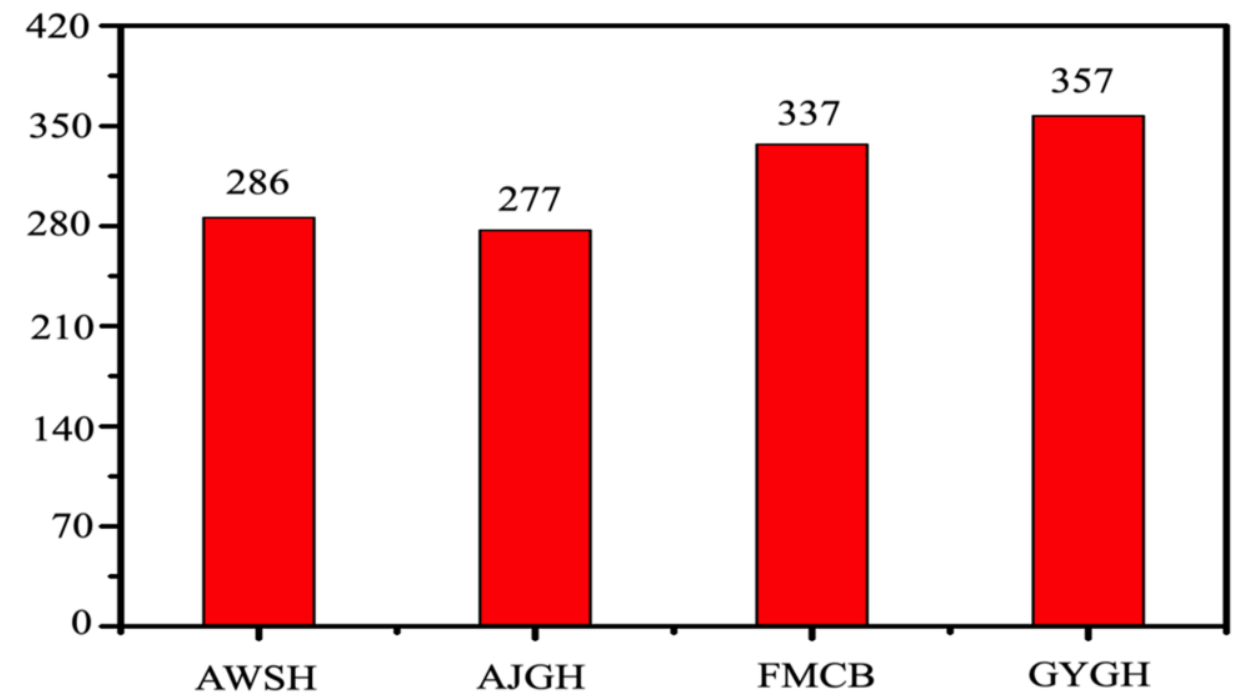

Figure 3. Total number of diabetes patients recorded in the four hospitals.

\subsection{Attributes Selection}

In our prediction assessment of diabetes mellitus prevalence, the data of 10 easily available attributes/variables, namely age, gender, GLU (glucose level of the patient), BMI (body mass index of the patient), HYP (hypertension status), HCD (history of cardiovascular disease), FDH (family history of diabetes), PEX (physical exercise), STW (work stress status), and DIT (diet of the patient, healthy and unhealthy). Out of 1257 records, 587 patient records were missing values in the body mass index, glucose level, hypertension, cardiovascular disease, work stress status, family diabetes experience, physical exercises, and diet lifestyles. Moreover, 389 records were removed from assessment dataset because of missing values in pre-diabetes status. Therefore, 281 records with 10 variables were used in the prediction analysis.

\subsection{Attribute Parameters}

The 10 features selected in this study were characterized as follows. Age and gender represented demographic characteristics. A patient's glucose level $(\mathrm{mmol} / \mathrm{L})$ has a relation with age and diet. 
Family history of diabetes was defined as any family member previously diagnosed by a physician as diabetic or pre-diabetic (Yes $=1, \mathrm{No}=0$ ). BMI was calculated as body weight divided by the square of height in meters and BMI $\geq 25$ was defined as overweight. History of cardiovascular disease or stroke was defined as the patient having been previously diagnosed with coronary heart disease or stroke by a surgeon (Yes $=1, \mathrm{No}=0$ ). Physical exercise indicated whether the patient engaged in exercise $($ Yes $=1$, No $=0)$. Work stress was measured to the patient's subjective impression (Yes $=1$, No $=0$ ). Diet was measured as a balanced and unbalanced diet (Yes $=1$, No $=0)$. HYP was defined in three ways: first, a systolic BP (blood pressure) $\geq 140 \mathrm{mmHg}$; second, medication for BP control; and third, diastolic $\mathrm{BP} \geq 90 \mathrm{mmHg}$.

\subsection{Data Mining Platform}

Waikato Environment for Knowledge Analysis (Weka/v 3.9.2) was used for the preprocessing and classification assessment of diabetes mellitus by updated plugins of the Kmean clustering algorithm to assign the class to the dataset of 10 variables for testing as positive and negative status (positive mean diabetes and negative mean normal status) [34]. The positive patients were declared as high in diabetes status after assessment and negative as normal for the initial screening by proper forecast assessment. The advantage of using Weka is the avoidance of overfitting and unnecessary complexity.

In addition, Rule algorithms (PART and Decision table) were adopted for accurate measurements. Moreover, the logistic regression was utilized on the assessment of classification to forecast diabetes prevalence for clinical implications.

After data preprocessing, the final dataset included 281 patient records with males and females and 11 attributes. The population sampling included patients with diabetes mellitus status Type 1 (non-insulin dependent), Type 2 (insulin-dependent), and gestational diabetes. The 11 attributes included 10 as input attributes and the one as the target attribute. The target attribute consisted of two classes: one class obtained the diagnosis of diabetes tested positive and the second class was tested negative by the Kmean finding within the clusters that are more related to each other at the significance level of 0.05 [35].

Kmean is a typical distance-based cluster algorithm and its distance is measured on similarities. The process steps of the Kmean are to measure the distance between each object and the centers of the cluster by Equations (1)-(3), as follows:

$$
\begin{gathered}
S_{i}^{(t)}=\left\{\forall j, 1 A j A k X_{p}:\left\|X_{p}-m_{i}^{t}\right\|^{2} \leq\left\|X_{p}-m_{j}^{t}\right\|^{2} \forall j, 1 \leq j \leq k\right\} \forall j, 1 A j A k, \\
m_{i}^{t+1}=\frac{1}{\left|S_{i}^{(t)}\right|} \sum_{x_{j} \in S_{i}^{(t)}} X_{j}, \\
J=\sum_{j=1}^{k} \sum_{i=1}^{n}\left\|x_{i}^{(j)}-c_{j}\right\|^{2},
\end{gathered}
$$

where $\mathrm{n}$ is the number of data points in the $i$ clusters, $\mathrm{k}$ is the number of cluster centers, and $\left\|x_{i}^{(j)}-c_{j}\right\|$ represents the Euclidean distance between $x_{i}^{(j)}$ and $c_{j}$. In addition, the Kmean clustering algorithm is composed of the following steps.

(i) Place the K points into the considerable space as represented by the objects that are being clustered, which indicate the initial group of centroids.

(ii) Properly assign each object to the group that undoubtedly possesses the most adjacent centroid.

(iii) After assigning all objects, recalculate the prominent position of the $\mathrm{K}$ centroid.

(iv) Repeat the second and third step until the centroids are not able to shift significantly more. This efficiently produces the possible separation of group objects, which can accurately calculate the matrix to be minimized by Equation (4). 


$$
\operatorname{argmin}_{c_{j} \in C} \operatorname{dist}\left(c_{i}, x\right)^{2}
$$

\subsection{Rules Classification}

The machine learning algorithms PART and Decision table were utilized for the classification of the dataset with a 10-fold cross-validation assessment. PART classifications are projected as discrete rules to conquer the rule methods of any dataset and generate a rule set for a better understanding of the decision list. In addition, PART works with a combination of C4.5 and Ripper [36]. The paramount leaf in the rules assessment was generated by the fractional C 4.5 decision tree repetitions. It compares the data to the rules of each list, and vice versa, and assigns the items accordingly.

The decision table summarizes the testing dataset and compares it with the training dataset generated. In addition, it classifies the unknown dataset samples by the Wrapper method, which helps to reduce the unknown values and produce better results with higher accuracy and minimal error rates [37]. The first attribute in the rule tree is the most informative node, which is measured by Equations (5) and (6):

$$
\begin{gathered}
I_{A}=E(D)-\sum_{i=1}^{k} \frac{\left|D_{i}\right|}{|D|} E\left(D_{i}\right), \\
E(X)=-\sum_{i=1}^{m} \frac{\operatorname{count}\left(c_{i}, x\right)}{|x|} \cdot \log \frac{\operatorname{count}\left(c_{i}, x\right)}{|x|} .
\end{gathered}
$$

The parameter selected for the PART classifier was 100 as the batch size with false in binary splits by a confidence interval of $0.25 \%$. The number of objects was set as 2 , decimal number places as 2 , fold number as 3 , error pruning as false, and seeds value as 1 . In addition, the parameters for the Decision table were 100 as the batch size with a cross value was 1 and the number of decimal places was 2 with the best first in search results.

\subsection{Kappa Statistics}

Kappa statistics have the consistency of frequent testing, which provides extended facts about data collection in the research that is correct for variable measurements. It compares the model results with the randomly generated classification. We adopted kappa stats measures based on values between 0 and 1 as in Equations (7)-(9) where the value 0 is invalid and 1 is the expected effect of the assessment. Furthermore, kappa stats indicate the consistency of assessment.

$$
\begin{gathered}
K=[P(A)-P(E)] /[1-P(E)] \\
P(A)=[(T P+T N) / N] \\
P(E)=\left[(T P+F N) *(T P+F P) *(T N+F N) / N^{2}\right.
\end{gathered}
$$

\subsection{Logistic Regression Forecasting}

Logistic regression was implemented on the classification outcomes with the primary objective to define the initial screening for disease diagnosis and prediction [38]. In most cases, the variables of the logistic regression work to solve the two-way binary classifications. It predicts the continuous values to maintain the sensitivity in the numbers field where the values are 0 and 1 . The value 1 is assigned only if the value is greater than the threshold (value $>$ threshold); otherwise, it will be 0 . Hence, the range of output works in the logistic regression is between 0 and 1 with the addition of the sigmoid function layers measured by Equations (10)-(13):

$$
\begin{gathered}
P=\alpha+\beta_{1} X_{1}+\beta_{2} X_{2}+\ldots+\beta_{m} X_{m}, \\
\sigma(x) \frac{1}{1+e^{-x}} \in[0,1],
\end{gathered}
$$




$$
\begin{gathered}
\operatorname{Pr}(Y=+1 \mid X) \sim \beta . X, \\
\operatorname{Pr}(Y=-1 \mid X)=1-\operatorname{Pr}(Y=+1 \mid X) .
\end{gathered}
$$

It consists of a positive and a negative group of values. The variable $X$ will be assigned to the $\beta$ coefficient values, which represent the weight. $Y$ indicates the patients with diabetes. The variations between the values $X$ and $Y$ occur on the basis of weight.

The parameters selected for the logistic regression forecast was 1 for a number of time units. The confidence interval was set at $0.95 \%$. The M 5 method was chosen for attribute selection with a batch size of 100 , and the ridge was set as $1.0 \mathrm{E}-8$. After accurately setting up, it is easy to predict the outcome of positive or negative. The sigmoid function $\sigma(\mathrm{x})$ proposition is described as follows:

Proposition 1. A function $f:(0,1) \rightarrow R$ is absolutely a monotone on $(0,1)$ if and only if it possesses a power series expansion with non-negative coefficients, converging for $0<x<1$.

Proof. If $(f)$ function is completely monotone in $(0,1)$, then the power series expansion of $(f)$ function in $(0,1)$ has to be alternating because $(-1)^{k} f^{k} \geq 0$. On the other hand, consider an alternating power series of function $f(x)$ converging for all $0<x<1$ and its derivatives by Equations (14)-(16):

$$
\begin{gathered}
f(x)=a_{0}-a_{1} x+a_{2} x^{2}-a_{3} x^{3} \ldots a_{i} \geq(0<x<1), \\
(-1) f^{1}(x)=a_{1}-2 a_{2} x+3 a_{3} x^{3}+\ldots, \\
f^{2}(x)=2 a_{2}-6 a_{3} x+\ldots
\end{gathered}
$$

\section{Results}

A total of 281 diabetes patients were evaluated; 121 (43.06\%) were male and 160 (56.93\%) were female. Among the 281 records, 256 (91.10\%) were not dependent on insulin (Type 1), 14 (4.98\%) were Gestational, and 11 (3.91\%) were insulin dependent (Type 2). Initially, the dataset was divided into a 20:80 ratio for conducting training and testing. After training the machine, a 10-fold cross-validation technique was implemented on an experimental platform of Weka for better assessment of the classification. The dataset was divided into 10 samples. Each sample was utilized as validation data from the retention process, while the remaining nine samples served as the training data. This process was performed 10 times. The advantage of this process is the reduction in the error ratio and bias correlation by random sampling.

\subsection{Measurements}

Initially, the PART rule classifier was tested on the dataset to measure the classification accuracy with the seed of random numbers selected for XVal. The percentage was 1, the confidence factor was $0.25 \%$, the minimum number of objects was 2 , and the number of folds was set to 3 . After loop tests, the average accuracy of the final result was $99.28 \%$. Secondly, the same measurement was tested on the Decision table rule classifier. The final result with an average accuracy of $98.22 \%$ was obtained in $0.77 \mathrm{~s}$. The subset value was $99.60 \%$, and the average error was $0.03 \%$. By employing the rule classification (PART and Decision table), good predictive rules were obtained for the patient's care. The outcomes in the initial phase were the most appropriate with a mean accuracy of $98.75 \%$; the error rate remained at $0.02 \%$.

The results obtained for the classification accuracy are presented in Table 1 along with the attribute details and the clustering instances for the classification. It is comprised of three sections. The first section discusses the details of the properties used for the Weka platform for assessment, with 281 patients describing their age limits by classification type and improving the evaluation of positive 
and negative tested weights. Additionally, it provides accurate information and average classification accuracy for PART and Decision table rule classifiers, including kappa statistics, mean error, true positive rate, false positive rate, accuracy, recall rate, F-measure, Matthew's correlation curve (MCC)., Receiver operating characteristics (ROC), Precision recall curve (PRC) area ratios, and the time it takes for a prediction analysis $[39,40]$.

Table 1. Diabetes type and the number of patients classified for the Rule assessment.

\begin{tabular}{|c|c|c|c|c|c|c|c|}
\hline \multirow{2}{*}{ Diabetes Type } & \multirow{2}{*}{$\begin{array}{l}\text { Patients } \\
(N=281)\end{array}$} & \multirow{2}{*}{ Age } & \multirow{2}{*}{ Weight } & \multirow{3}{*}{$\begin{array}{c}\text { “0” } \\
\text { Missing } \\
\text { Values }\end{array}$} & \multirow{2}{*}{ Attributes } & \multicolumn{2}{|c|}{ Class } \\
\hline & & & & & & $\mathbf{T} \_\mathbf{N}$ & T_P \\
\hline NID & 256 & \multirow{3}{*}{$>10<87$} & 256.0 & & \multirow{3}{*}{11} & \multirow{3}{*}{87} & \multirow{3}{*}{194} \\
\hline GTD & 14 & & 14.0 & & & & \\
\hline IND & 11 & & 11.0 & & & & \\
\hline \multicolumn{3}{|c|}{ Classification } & \multicolumn{3}{|c|}{ PART Rule \% } & \multicolumn{2}{|c|}{ Decision Table Rule \% } \\
\hline \multicolumn{8}{|c|}{ Total number of diabetes mellitus patients from age $>10$ and $<87(N=281)$} \\
\hline \multicolumn{8}{|c|}{$\begin{array}{ll}\text { - } & \text { From age } \leq 20=2 \text { patients } \\
\text { - } & \text { From age }>20 \text { and } \leq 40=58 \text { patients } \\
\text { - } & \text { From age }>40 \text { and } \leq 60=144 \text { patients } \\
\text { - } & \text { From age }>60 \text { and } \leq 80=76 \text { patients } \\
\text { - } & \text { From age }>80=1 \text { patient }\end{array}$} \\
\hline \multicolumn{3}{|c|}{ Accuracy } & \multicolumn{3}{|c|}{99.28} & \multicolumn{2}{|c|}{98.22} \\
\hline \multicolumn{3}{|c|}{ Kappa statistics } & \multicolumn{3}{|c|}{0.98} & \multicolumn{2}{|c|}{0.96} \\
\hline \multicolumn{3}{|c|}{ Mean absolute error } & \multicolumn{3}{|c|}{0.01} & \multicolumn{2}{|c|}{0.03} \\
\hline \multicolumn{3}{|c|}{ True positive rate } & \multicolumn{3}{|c|}{0.99} & \multicolumn{2}{|c|}{0.98} \\
\hline \multicolumn{3}{|c|}{ False positive rate } & \multicolumn{3}{|c|}{0.01} & \multicolumn{2}{|c|}{0.01} \\
\hline \multicolumn{3}{|c|}{ Precision } & & 0.99 & & & \\
\hline & Recall & & & 0.99 & & & \\
\hline & -Measure & & & 0.99 & & & \\
\hline & MCC & & & 0.98 & & & \\
\hline & OC area & & & 0.99 & & & \\
\hline & RC area & & & 0.99 & & & \\
\hline Time tak & to build the & del & & $0.10 \mathrm{~s}$ & & & \\
\hline & age accuracy & & & 99.28 & & & \\
\hline Mean & verage accura & & & & $98.75 \%$ & & \\
\hline Values & Counts & Ratio & Clust & y Class & Clust & by Diab & \\
\hline & $\left(\mathrm{N}^{\prime}=281\right)$ & & $\mathbf{T} \_\mathbf{N}$ & $\mathbf{T}+\mathbf{P}$ & NID & GTD & IND \\
\hline 0 & 138 & $49 \%$ & 47 & 91 & 128 & 7 & 3 \\
\hline 1 & 143 & $51 \%$ & 40 & 103 & 128 & 7 & $8^{1}$ \\
\hline
\end{tabular}

${ }^{1} \mathrm{NID}=$ not insulin dependent; GTD = gestational diabetes patients; IND = insulin dependent; MCC = Matthew's correlation curve; $\mathrm{ROC}=$ Receiver operating characteristics; $\mathrm{PRC}=$ Precision recall curve; $\mathrm{N}=$ number of patients; $\geq$ greater than; $\leq$ less than; $\%=$ percentage value; $T_{-} \mathrm{N}=$ tested negative; $\mathrm{T} \_\mathrm{P}=$ tested positive; Values = two clusters 0 and $1 ; N^{\prime}=$ total number of classified patients.

The details of the cluster instance, as shown in Figure 4, was tested and classified as positive/negative. Out of 281 instances, $138(49.11 \%)$ were classified as the 0 cluster instance, among them $47(16.72 \%)$ were tested as negative, and $91(32.38 \%)$ were tested as positive. One hundred and forty-three $(50.88 \%)$ were classified as a cluster 1 instance from which $40(14.23 \%)$ were tested as negative and $103(36.65 \%)$ were tested as positive. In the final assessment, 51\% were classified as positive and $49 \%$ instances as negative. The values of these classifications were used as input to the regression prediction phase. 


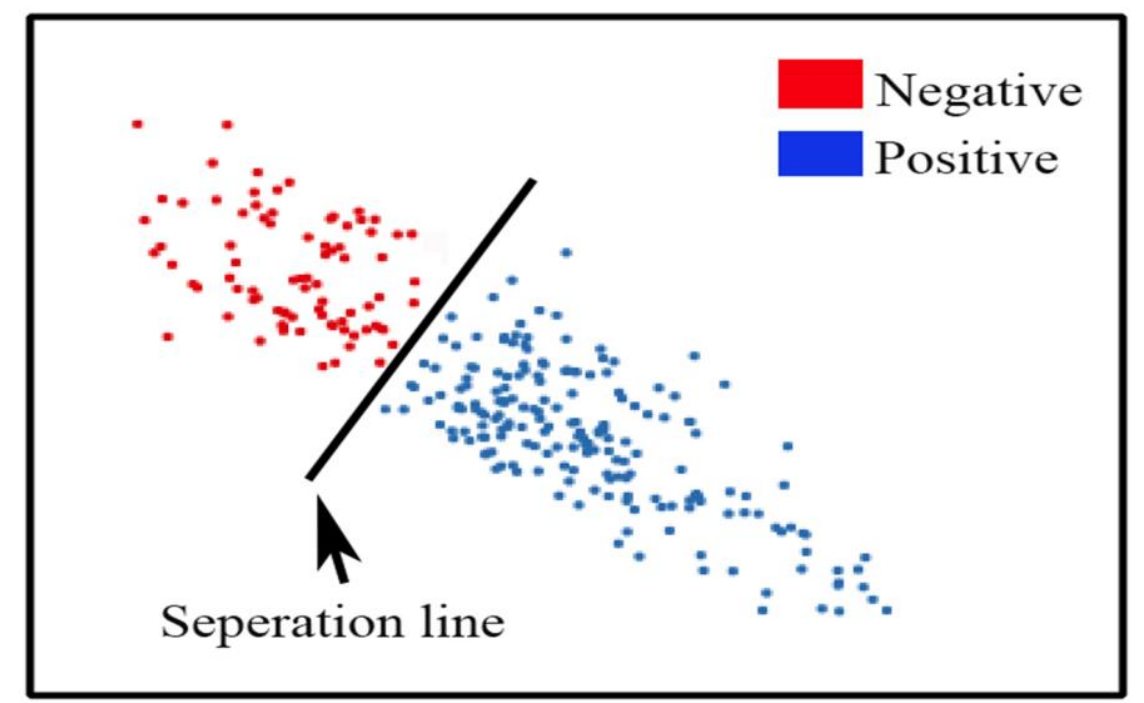

Figure 4. Evaluation of the Kmean clusters tested as positive and negative.

\subsection{Rule Forecast Assessment}

The predictive analysis represents the assessment for decision-making by determining the ratio of patient characteristics. The forecast analysis obtained in the study is graphically displayed in Figure $5 \mathrm{a}-\mathrm{g}$, and the 23 rules achieved through the rule classification measurements are described in Table 2 in terms of the patients' initial screening stage of healthcare.

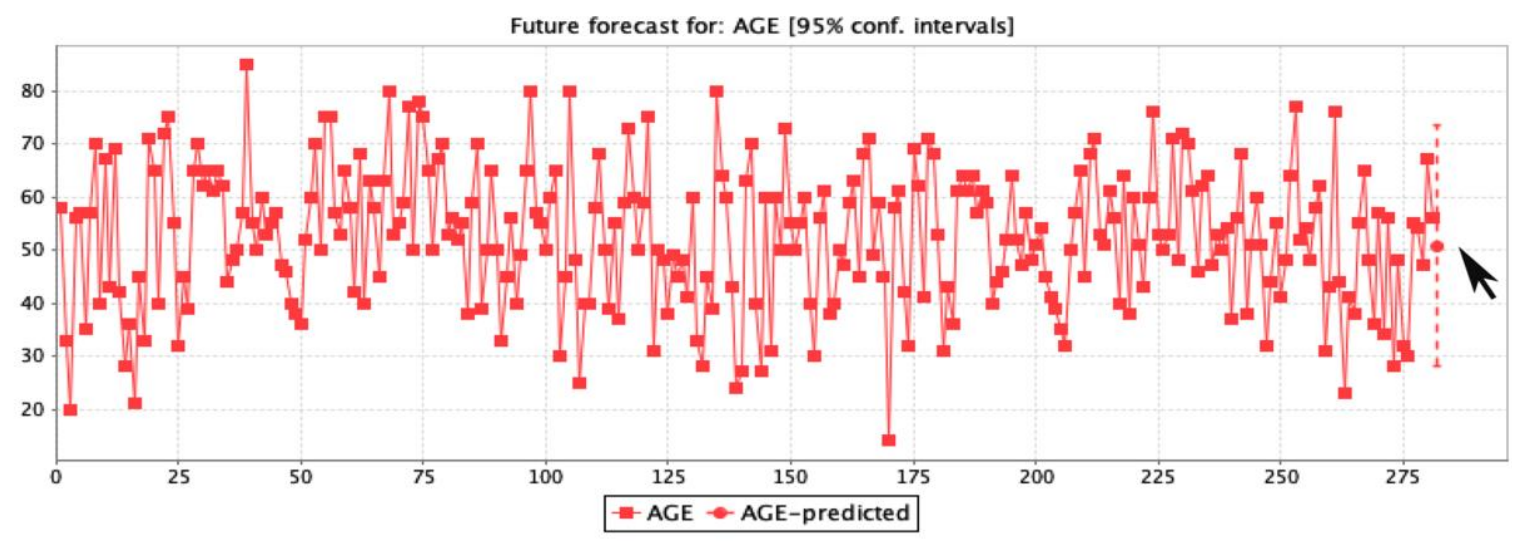

(a)

Figure 5. Cont. 


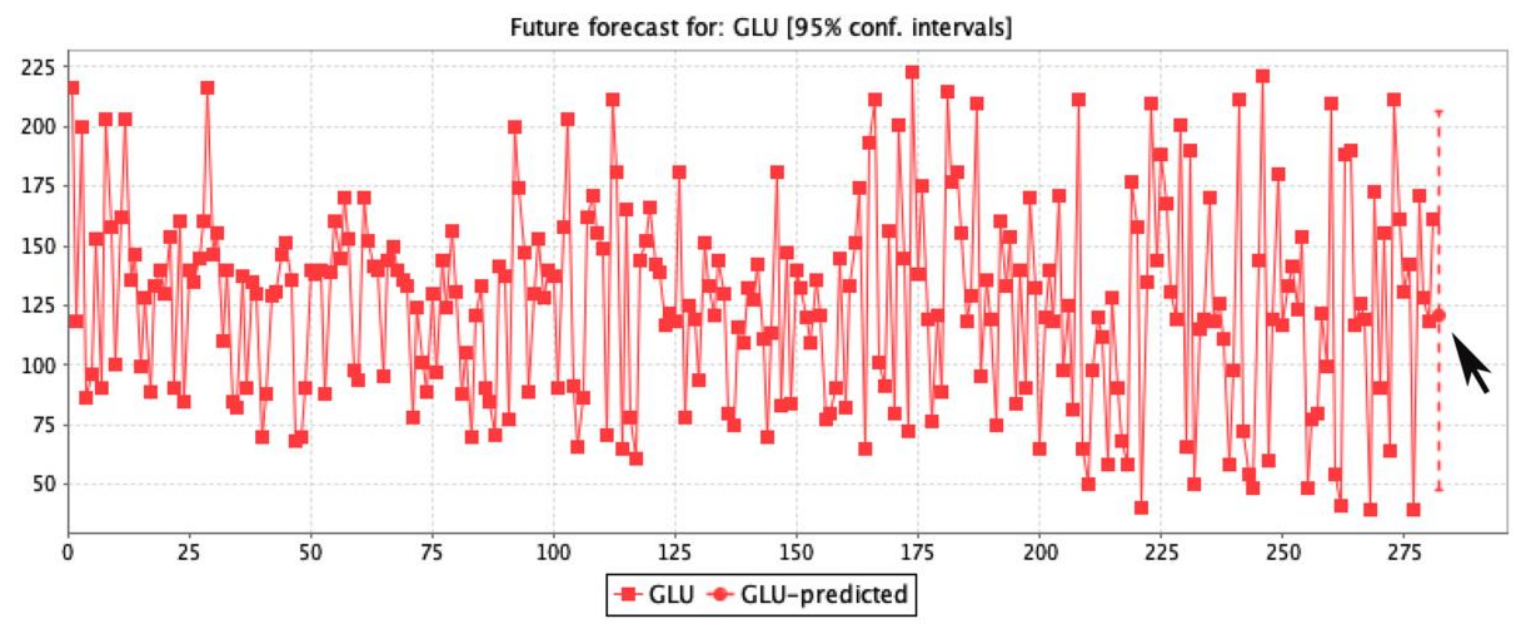

(b)

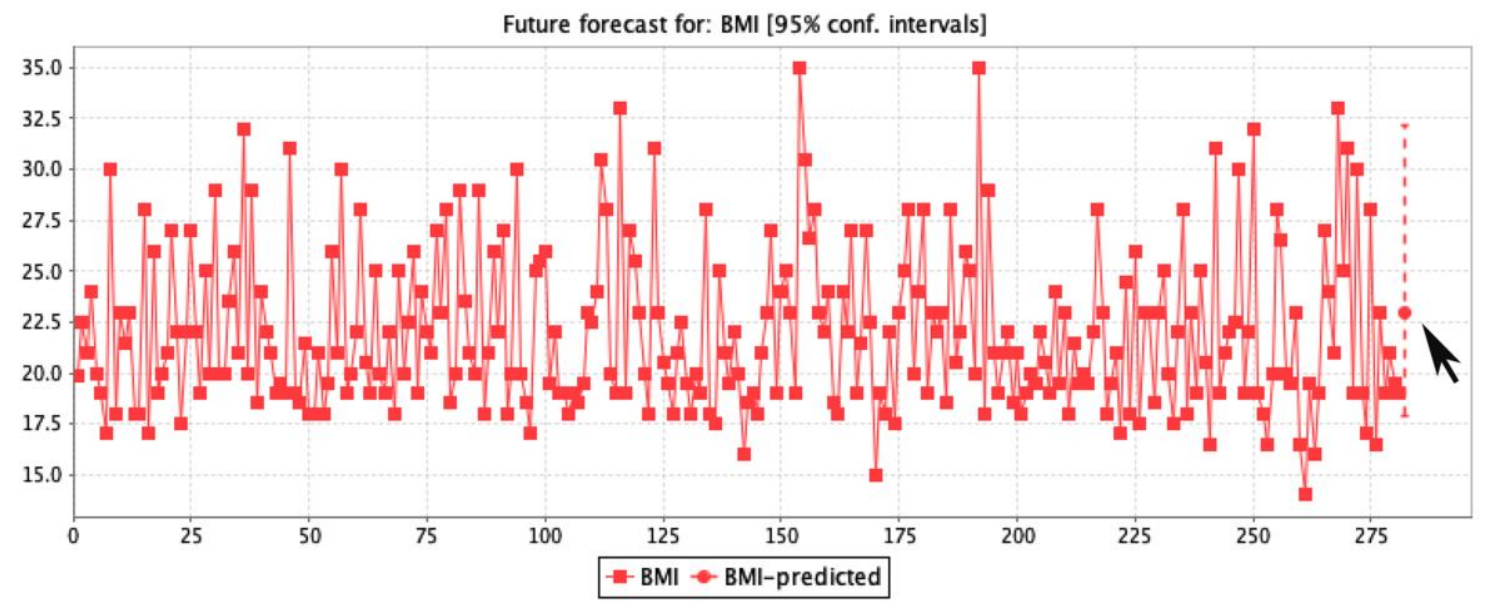

(c)

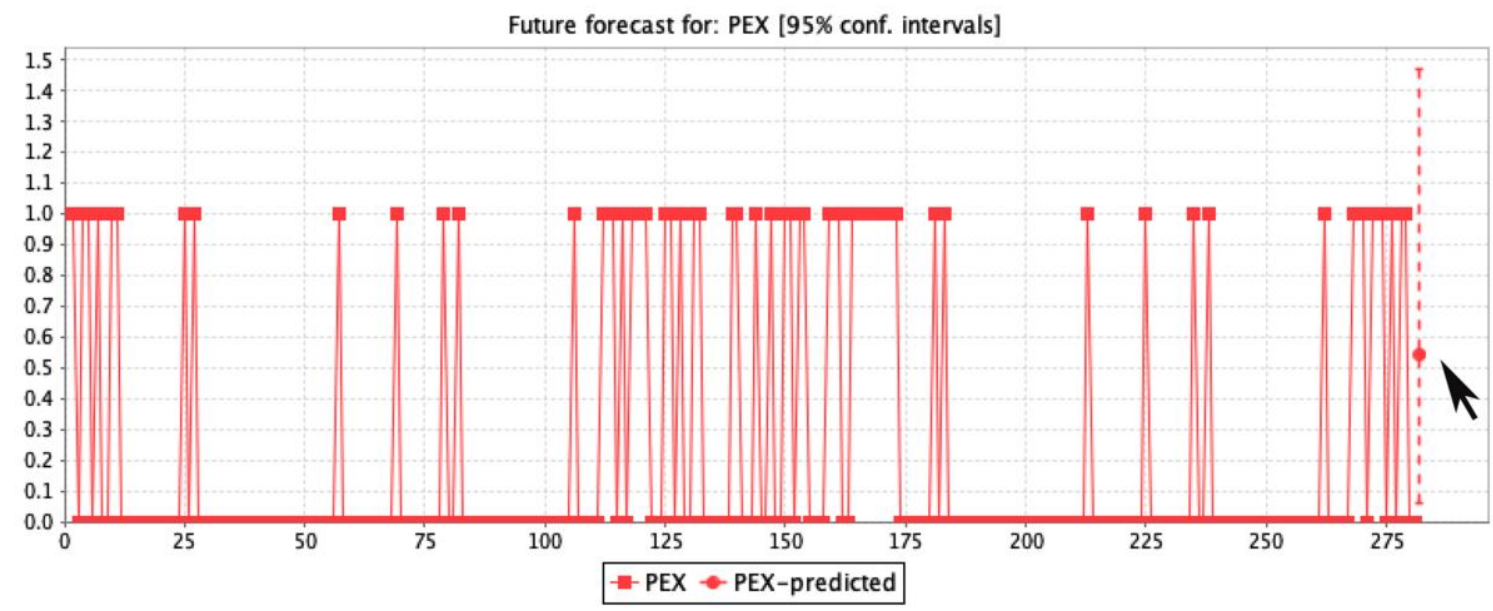

(d)

Figure 5. Cont. 


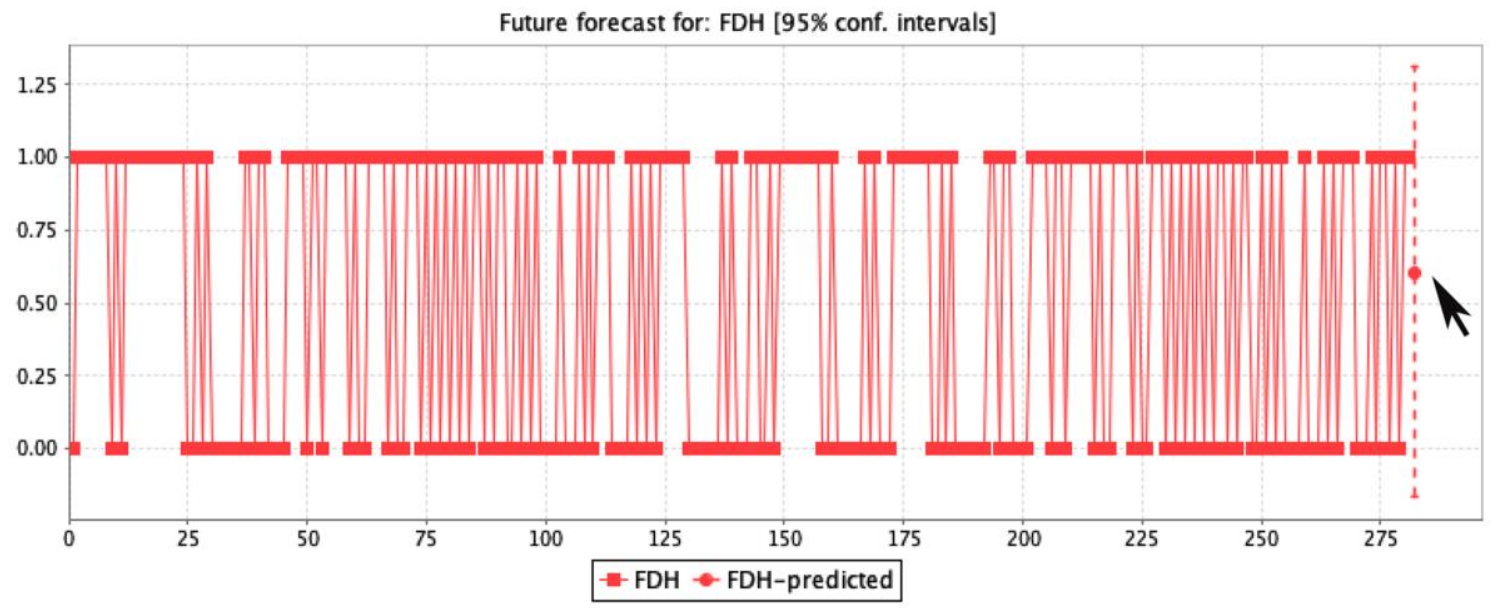

(e)

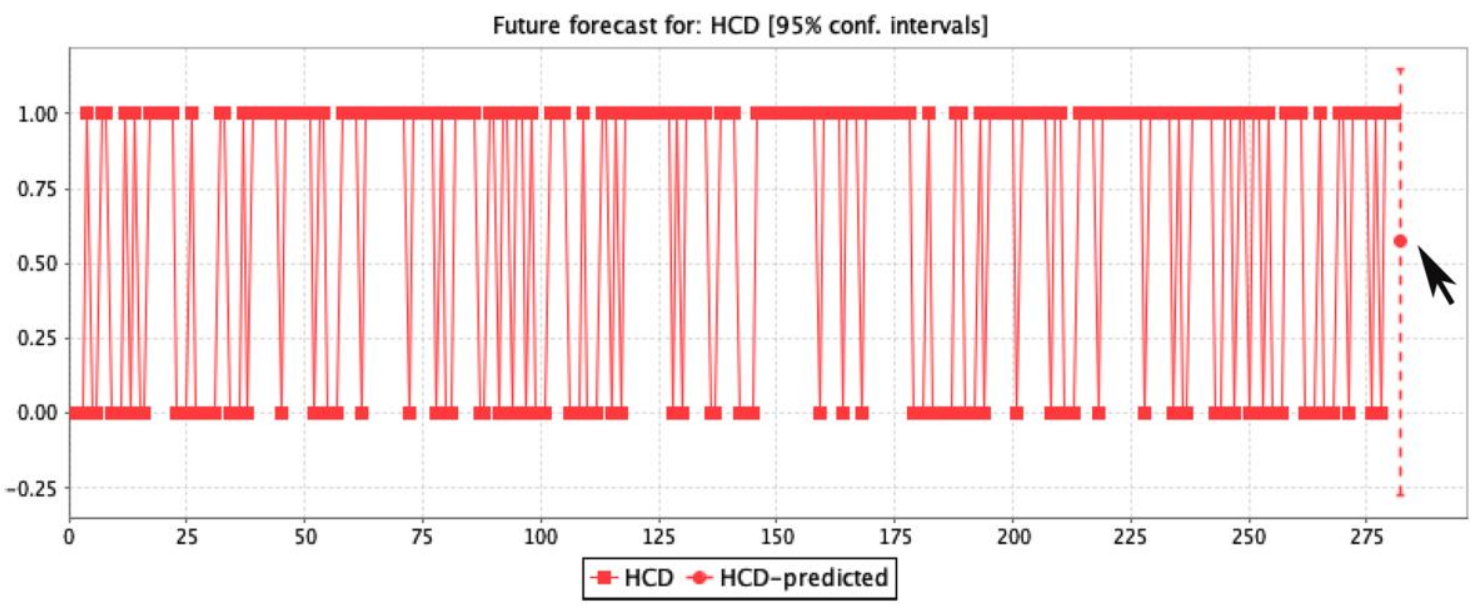

(f)

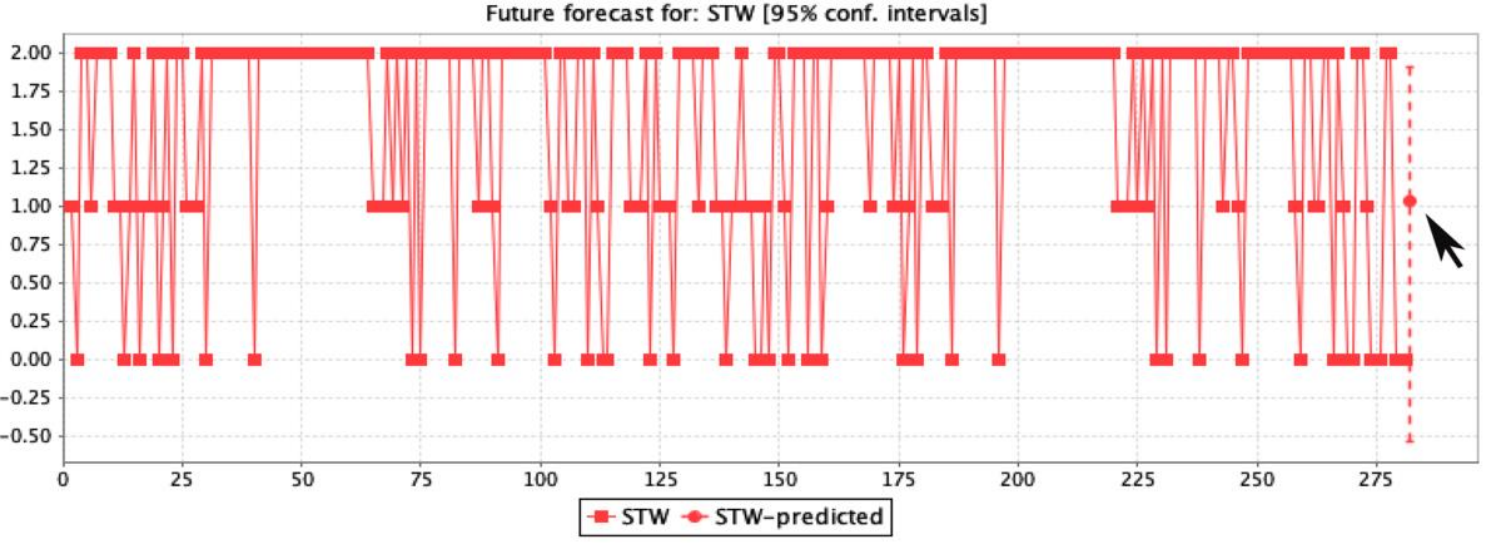

(g)

Figure 5. (a-g) The regression prediction assessment of the seven main features used for the analysis of clinical significance. 
Table 2. Twenty-three if-then rules achieved from the classification analysis.

\begin{tabular}{|c|c|}
\hline \multicolumn{2}{|r|}{ Twenty-Three If-Then Rules Extracted from the Assessment Are: } \\
\hline Rule 1: & $\begin{array}{l}\text { IF the patient's glucose level is }(>101) \text {; THEN the patient is classified as tested } \\
\text { positive with diabetes. }\end{array}$ \\
\hline Rule 2: & $\begin{array}{l}\text { IF the patient's glucose level is }(>72) \text {; THEN the patient is classified as tested } \\
\text { positive for diabetes, but the patient has to screen through the second stage test. }\end{array}$ \\
\hline Rule 3: & $\begin{array}{l}\text { IF the patient's blood pressure is }(\leq 100) \text {; THEN the patient is classified as tested } \\
\text { negative for diabetes but this case also depends on the glucose level of the patient, } \\
\text { which takes patients for screening of the second stage. }\end{array}$ \\
\hline Rule 4: & $\begin{array}{l}\text { IF the patient's blood pressure is }(<100) \text {; THEN the patient is classified as tested } \\
\text { negative for diabetes but the patient has to go through the second stage of } \\
\text { screening. }\end{array}$ \\
\hline Rule 5: & $\begin{array}{l}\text { IF the patient's (age } \leq 49) \text { and }(B M I \leq 25) \text { and the patient also has no diabetes in } \\
\text { their family history; THEN the patient is classified as tested negative for diabetes. }\end{array}$ \\
\hline Rule 6: & $\begin{array}{l}\text { IF the patient's (age } \leq 34) \text { and }(B M I>25) \text { and the patient also has no diabetes in } \\
\text { their family history and patient's diet is unbalanced; THEN the patient is } \\
\text { classified as tested negative for diabetes. }\end{array}$ \\
\hline Rule 7: & $\begin{array}{l}\text { IF the patient's age is from }(35 \leq 49) \text { and }(\mathrm{BMI}>25) \text { and the patient also has no } \\
\text { diabetes in their family history and the patient's diet is unbalanced and the } \\
\text { patient is without physical exercise; THEN the patient is classified as tested } \\
\text { positive for diabetes. }\end{array}$ \\
\hline Rule 8: & $\begin{array}{l}\text { IF the patient's age is from }(35 \leq 49) \text { and }(\mathrm{BMI}>25) \text {, and the patient also has no } \\
\text { diabetes in their family history, the patient's diet is unbalanced, and the patient is } \\
\text { with physical exercise but has no history of cardiovascular disease; THEN the } \\
\text { patient is classified as tested negative for diabetes. }\end{array}$ \\
\hline Rule 9: & $\begin{array}{l}\text { IF the patient's age is from }(35 \leq 49) \text { and }(\mathrm{BMI}>25) \text {, and the patient also has no } \\
\text { diabetes in their family history, the patient's diet is unbalanced, and the patient is } \\
\text { with physical exercise but has no history of cardiovascular disease; THEN the } \\
\text { patient is classified as tested positive for diabetes. }\end{array}$ \\
\hline Rule10: & $\begin{array}{l}\text { IF the patient's age is }(\leq 49) \text { and }(\mathrm{BMI}>25) \text {, and the patient also has no diabetes in } \\
\text { their family history and the patient's diet is balanced; THEN the patient is } \\
\text { classified as tested negative for diabetes. }\end{array}$ \\
\hline Rule11: & $\begin{array}{l}\text { IF the patient's age is }(\leq 49) \text { and }(\mathrm{BMI} \leq 25) \text {, and the patient also has diabetes in } \\
\text { their family history; THEN the patient is classified as tested negative for diabetes. }\end{array}$ \\
\hline Rule12: & $\begin{array}{l}\text { IF the patient's age is }(\leq 49) \text { and }(B M I>25) \text {, and the patient also has diabetes in } \\
\text { their family history; THEN the patient is classified as tested positive for diabetes. }\end{array}$ \\
\hline Rule13: & $\begin{array}{l}\text { IF the patient's age is }(>49) \text { and }(\mathrm{BMI} \leq 25) \text {, and the patient also has a high work } \\
\text { stress but no diabetes in their family history; THEN the patient is classified as } \\
\text { tested negative for diabetes. }\end{array}$ \\
\hline Rule14: & $\begin{array}{l}\text { IF the patient's age is }(>49) \text { and }(\mathrm{BMI}>25) \text {, and the patient also has a high work } \\
\text { stress but no diabetes in their family history; THEN the patient is classified as } \\
\text { tested positive for diabetes. }\end{array}$ \\
\hline Rule15: & $\begin{array}{l}\text { IF the patient's age is }(>49) \text { and the patient has a high work stress, and also has } \\
\text { diabetes in their family history; THEN the patient is classified as tested positive } \\
\text { for diabetes. }\end{array}$ \\
\hline Rule16: & $\begin{array}{l}\text { IF the patient's age is }(>49) \text { and }(\mathrm{BMI}>25) \text {, and the patient's work stress is low } \\
\text { and also has no diabetes in their family history but their diet is unbalanced; } \\
\text { THEN the patient is classified as tested positive for diabetes. }\end{array}$ \\
\hline Rule17: & $\begin{array}{l}\text { IF the patient's age is }(>49) \text { and }(\mathrm{BMI}>25) \text {, and the patient has no diabetes in } \\
\text { their family history and has a balanced diet; THEN the patient is classified as } \\
\text { tested negative for diabetes. }\end{array}$ \\
\hline Rule18: & $\begin{array}{l}\text { IF the patient's age is }(>49) \text { and }(\mathrm{BMI}>25) \text {, and the patient's work stress is low } \\
\text { but they have diabetes in their family history; THEN the patient is classified as } \\
\text { tested positive for diabetes. }\end{array}$ \\
\hline
\end{tabular}


Table 2. Cont.

\begin{tabular}{|c|c|}
\hline \multicolumn{2}{|r|}{ Twenty-Three If-Then Rules Extracted from the Assessment Are: } \\
\hline Rule19: & $\begin{array}{l}\text { IF the patient's age is }(>49) \text { and }(\mathrm{BMI} \leq 25) \text {, and the patient has a low or medium } \\
\text { work stress with hypertension and also their food is not balanced; THEN the } \\
\text { patient is classified as tested positive for diabetes. }\end{array}$ \\
\hline Rule20: & $\begin{array}{l}\text { IF the patient is male with age }(>49) \text { and }(\mathrm{BMI} \leq 25) \text {, and the patient has a low or } \\
\text { medium work stress without hypertension and also their food is not balanced but } \\
\text { they have diabetes in their family history with cardiovascular disease; THEN the } \\
\text { patient is classified as tested positive for diabetes. }\end{array}$ \\
\hline Rule21: & $\begin{array}{l}\text { IF the patient is male with age }(>49) \text { and }(\mathrm{BMI} \leq 25) \text {, and the patient has a low or } \\
\text { medium work stress without hypertension and their diet is not balanced, and } \\
\text { they have cardiovascular disease history in their family; THEN the patient is } \\
\text { classified as tested negative for diabetes. }\end{array}$ \\
\hline Rule22: & $\begin{array}{l}\text { IF the patient is female with age }(>49) \text { and }(\mathrm{BMI} \leq 25) \text {, and the patient has a low } \\
\text { or medium work stress without hypertension and their diet is not balanced; } \\
\text { THEN the patient is classified as tested negative for diabetes. }\end{array}$ \\
\hline Rule23: & $\begin{array}{l}\text { IF the patient's age is }(>49) \text { and }(\mathrm{BMI} \leq 25) \text {, and the patient has a low or medium } \\
\text { work stress with balanced diet; THEN the patient is classified as tested negative } \\
\text { for diabetes. }\end{array}$ \\
\hline
\end{tabular}

The prediction assessment by logistic regression used in this study for clinical significance was analyzed by the confidence interval of $0.95 \%$. The patient features used were age, blood glucose, body mass index, physical exercise, family history of diabetes, family cardiovascular history, and work stress by the M5 method in regression. The results of the forecast prediction for diabetes mellitus patients on the age feature show that patients up to 51 years could have a high death risk if the ratio of other features include a glucose level of $120.45 \mathrm{mmol} / \mathrm{L}, \mathrm{BMI} \geq 23$, physical exercise between 0.5 to 0.6 , family diabetes history of 0.6 , cardiovascular stroke history of 0.61 , and a work-stress ratio count of 1.08 .

\section{Discussion}

In this study, a machine-learning technique was instigated on a data-mining platform with a dataset of 281 patients suffering from diabetes. The data was collected only from Nigeria for the assessment of diabetes mellitus prevalence by determining two rule classifiers (PART and Decision tables) on 10 non-invasive and easily accessible medical attributes/variables. They include age (age of the patient), gender (male and female), glucose level of the patient, body mass index of the patient, hypertension, history of cardiovascular disease, family history of diabetes, physical exercise, stress of work, and diet of the patient (healthy and unhealthy) to accurately measure diabetes mellitus ratio for rapid and precise screening of patients suffering with diabetes mellitus status along with other chronic disease symptoms.

Initially, during the assessment on the data mining platform (Weka), the dataset was divided into two parts for training and testing in a 20:80 percent ratio. Twenty percent of the training data was used to train the machine and assess the outcome. Whereas, 80 percent of the data was used for testing. Furthermore, a complete dataset of 281 patients was analyzed on the experimental mode of Weka for the final assessment of both classifiers together. The results of the Rule classification show the mean accuracy of $98.75 \%$ with an error rate of $0.02 \%$. In addition, the mean kappa stats were $0.97 \%$, true positive rate remained $0.97 \%$, false positive rate $0.01 \%$, precision $0.98 \%$, recall $0.98 \%$, F-matrix $0.98 \%$, MCC $0.97 \%$, ROC area ratio $0.99 \%$, and PRC area ratio $0.99 \%$.

The outcomes of the non-invasive medical features used in this study indicate this assessment can successfully help to predict the patients of diabetes and pre-diabetes without the need for preliminary laboratory tests. In addition, the 23 rules generated during the assessment clearly show the main features of individuals with diabetes. Therefore, this study raises the prediction that age is the underlying and root variable, followed by a family history of diabetes, body mass index, gender, 
work stress, physical exercise, diet lifestyle, hypertension, and cardiovascular family history. These implementations are useful for substantial epidemiological threats and low socioeconomic status regions around the world, such as Africa and other developing states.

The key strength of this study is its use of a unique approach to both classifiers with logistic regression assessment to identify and forecast diabetes mellitus prevalence. Moreover, the use of realistic health records collected from the four principal hospitals in the developing country of Nigeria where the prevalence proportion of diabetes in men and women is high and explicitly mentioned in the literature study. Hence, patients with diabetes mellitus can be screened by 23 generated rules. Diabetes mellitus can be controlled through organizing appropriate educational programs in developing countries to govern the widespread growth of diabetes mellitus. This can help people reduce the burden of health hitches through awareness-raising activities. The classification assessment proposed in this paper was set to test other well-known machine learning algorithms by the same data to evaluate and compare classification accuracy results. Table 3 and Figure 6 clearly show that PART and Decision table rule classifiers have been successful in clinically meaningful research.

Table 3. The rule classification average precision is compared to other machine learning classifiers based on the same dataset.

\begin{tabular}{|c|c|c|c|}
\hline Method & Accuracy\% & & Mean \% \\
\hline PART rule & 99.28 & & \\
\hline Decision table rule & 98.22 & & $98.75 \%$ \\
\hline MLP & & 73.82 & \\
\hline Discrim & & 77.54 & \\
\hline Logdisc & & 78.22 & \\
\hline KNN & & 94.29 & \\
\hline Logistic & & 85.35 & \\
\hline BayesNet & & 74.76 & \\
\hline NaïveBayes & & 76.35 & \\
\hline Random Forest & & 76.66 & \\
\hline LogitBoost & & 93.93 & \\
\hline $\mathrm{J} 48$ & & 98.17 & \\
\hline SGD & & 76.62 & \\
\hline $\mathrm{SMO}$ & & 77.26 & \\
\hline ANN & & 89.84 & \\
\hline $\mathrm{RBF}$ & & 75.71 & \\
\hline FCM & & $94.78^{1}$ & \\
\hline
\end{tabular}

${ }^{1}$ It comprehensively compares the proposed classification results with the other machine learning classifiers on the same dataset. 


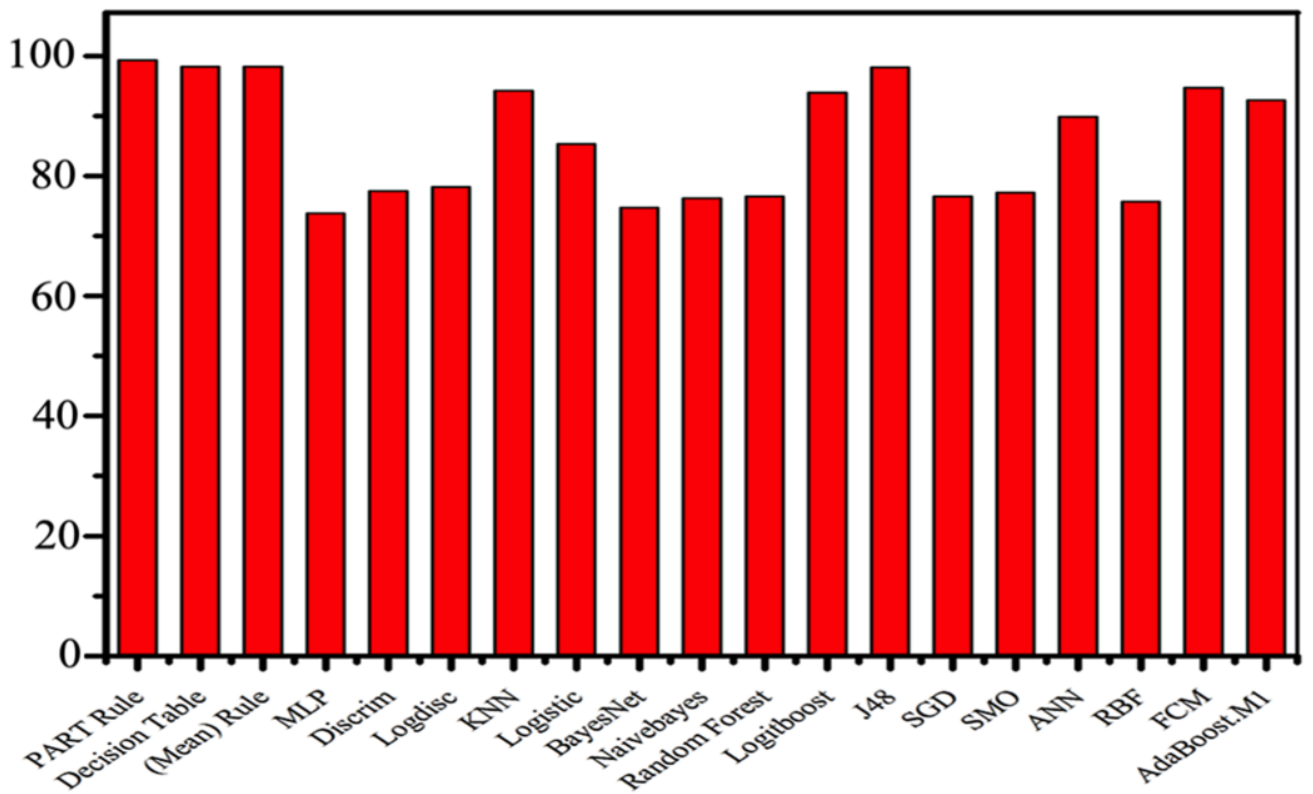

Figure 6. Comparisons of the rule classifier with other machine learning classifiers.

\section{Limitation}

The dataset was divided into a 20:80 percentages. Initially, 20\% was utilized for machine training and $80 \%$ were used for testing. Furthermore, 100\% with 281 instances were used in performing experiments on Weka to achieve the mean accuracy of both classifiers. This assessment study holds two limitations. One comprises a division of training and testing data for the meta-dataset and the second is the time taken to test the metadata for classification. If the metadata is analyzed on the same platform, the processing time can increase. However, it depends on the type of dataset used, the number of seeds input, and the number of experiments performed for acquiring the desired results.

\section{Conclusions}

This study implements the machine learning rule classifiers (PART and Decision table) on a data mining platform to identify possible diabetes and pre-diabetes in the initial clinical screening of a patient through logistic regression forecast assessment analysis. Two hundred and eighty-one diabetes mellitus patients have been analyzed with 10 easily available non-invasive medical features collected from four main hospitals located in northwestern Nigeria. The classification assessment accuracy was $98.75 \%$ and it was achieved through a set of 23-decision screening rules that can successfully influence accurate initial clinical screening of diabetes mellitus and pre-diabetes patients.

Additionally, the obtained Rules classified the most considerable risks and suggest that diabetes prevention and education programs can be applied in targeted community interventions. The study helps in the initial diagnosis of diabetes and reduces healthcare organization problems. Therefore, such a study is found extremely significant for the states and regions with extreme epidemic risk ratios and low socioeconomic status across the globe.

Author Contributions: Writing-Original draft preparation, methodology, software, and formal analysis have been done by M.N.S.; conceptualization, validation, data curation, and visualization, has done by M.N.S., M.U.M.; supervision, resources, project administration, and funding acquisition has done by R.J.; investigation has done by M.N.S., S.T.C., J.A.; finally writing—review and editing has carefully done by M.N.S., S.T.C., J.A., A.J.V.

Funding: This research work has been supported by NSFC Natural Science Foundation of Hebei province under grant of No. 61572420, No. 61472341, and No. 61772449.

Acknowledgments: We express our appreciation to "Yanshan University, Qinhuangdao, China" for accompanying us in this research. 
Conflicts of Interest: The authors declare no conflict of interest.

\section{Nomenclature}

PART

F-measure

CI

MCC

DR

DM

T2DM

GLU

BMI

HYP

HCD

FDH

PEX

STW

DIT

LR
Projective adaptive response theory

Frequency matrix

Confidence interval

Matthews's correlation coefficient

Decision rules

Diabetes mellitus

Type 2 diabetes mellitus

Glucose level

Body mass index

Hypertension

History of cardiovascular disease

Family diabetes history

Physical exercise

Work stress

Diet

Logistic regression

\section{References}

1. Khoury, N.; Attal, F.; Amirat, Y.; Oukhellou, L.; Mohammed, S.; Khoury, N. Data-Driven Based Approach to Aid Parkinson's Disease Diagnosis. Sensors 2019, 19, 242. [CrossRef]

2. Melmed, S.; Polonsky, K.S.; Larsen, P.R.; Kronenberg, H. Williams Textbook of Endocrinology, 13th ed.; Elsevier: Amsterdam, The Netherlands, 2016.

3. Whiting, D.R.; Guariguata, L.; Weil, C.; Shaw, J. IDF Diabetes Atlas: Global estimates of the prevalence of diabetes for 2011 and 2030. Diabetes Res. Clin. Pract. 2011, 94, 311-321. [CrossRef]

4. Cho, N.H.; Shaw, J.E.; Karuranga, S.; Huang, Y.; da Rocha Fernandes, J.D.; Ohlrogge, A.W.; Malanda, B. IDF Diabetes Atlas: Global estimates of diabetes prevalence for 2017 and projections for 2045. Diabetes Res. Clin. Pract. 2018, 138, 271-281. [CrossRef] [PubMed]

5. Shamshirgaran, S.M.; Jorm, L.; Lujic, S.; Bambrick, H. Health related outcomes among people with type 2 diabetes by country of birth: Result from the 45 and Up Study. Prim. Care Diabetes 2019, 13, 71-81. [CrossRef] [PubMed]

6. Gan, D.; King, H.; Lefèbvre, P.; Mbanya, J.-C.; Silink, M.; Siminerio, L. Diabetes Atlas, 2nd ed.; Gent: Brussels, Belgium, 2015.

7. American Diabetes Association. 1. Improving Care and Promoting Health in Populations: Standards of Medical Care in Diabetes-2019. Diabetes Care 2019, 42, S7-S12. [CrossRef]

8. Tsobgny-Tsague, N.-F.; Lontchi-Yimagou, E.; Nana, A.R.N.; Tankeu, A.T.; Katte, J.C.; Dehayem, M.Y.; Bengondo, C.M.; Sobngwi, E. Effects of nonsurgical periodontal treatment on glycated haemoglobin on type 2 diabetes patients (PARODIA 1 study): A randomized controlled trial in a sub-Saharan Africa population. BMC Oral Health 2018, 18, 28. [CrossRef]

9. Lélis, V.-M.; Guzmán, E.; Belmonte, M.-V. A Statistical Classifier to Support Diagnose Meningitis in Less Developed Areas of Brazil. J. Med. Syst. 2017, 41, 145. [CrossRef] [PubMed]

10. Awad, S.F.; O'Flaherty, M.; Critchley, J.; Abu-Raddad, L.J. Forecasting the burden of type 2 diabetes mellitus in Qatar to 2050: A novel modeling approach. Diabetes Res. Clin. Pract. 2018, 137, 100-108. [CrossRef]

11. Choi, S.B.; Kim, W.J.; Yoo, T.K.; Park, J.S.; Chung, J.W.; Lee, Y.; Kang, E.S.; Kim, D.W. Screening for prediabetes using machine learning models. Comput. Math. Methods Med. 2014, 2014, 618976. [CrossRef] [PubMed]

12. Talaei-Khoei, A.; Wilson, J.M.; Kazemi, S.-F. Period of Measurement in Time-Series Predictions of Disease Counts from 2007 to 2017 in Northern Nevada: Analytics Experiment. JMIR Public Heal Surveill. 2019, 5, e11357. [CrossRef] 
13. Olivera, A.R.; Roesler, V.; Iochpe, C.; Schmidt, M.I.; Vigo, Á.; Barreto, S.M.; Duncan, B.B. Comparison of machine-learning algorithms to build a predictive model for detecting undiagnosed diabetes_ELSA-Brasil: Accuracy study. Sao Paulo Med. J. 2017, 135, 234-246. [CrossRef] [PubMed]

14. Sohail, M.N.; Jiadong, R.; Uba, M.M.; Irshad, M. A Comprehensive Looks at Data Mining Techniques Contributing to Medical Data Growth: A Survey of Researcher Reviews; Springer: Singapore, 2019; pp. 21-26.

15. Kaur, P.; Sharma, N.; Singh, A.; Gill, B. CI-DPF: A Cloud IoT based Framework for Diabetes Prediction. In Proceedings of the 2018 IEEE Annual Information Technology, Electronics and Mobile Communication Conference, Columbia, Canada, 3 November 2018; pp. 654-660.

16. Mahmud, S.M.H.; Hossin, M.A.; Ahmed, M.R.; Noori, S.R.H.; Sarkar, M.N.I. Machine Learning Based Unified Framework for Diabetes Prediction. In Proceedings of the 2018 International Conference on Big Data Engineering and Technology (BDET 2018), Chengdu, China, 27 August 2018; pp. 46-50.

17. Srikanth, P.P.; Nilofer, V.; Siddiqui, I.; Dasari, P.; Ambica, B.; Venkata, V.B.V.E. Characteristic evaluation of diabetes data using clustering techniques. Int. J. Comput. Sci. Netw. Secur. 2008, 8, 244-251.

18. Okpor, M.D. Prognostic Diagnosis of Gestational Diabetes Utilizing Fuzzy Classifier. Int. J. Comput Sci. Netw. Secur. 2015, 15, 44.

19. Humayun, A.; Niaz, M.; Umar, M.; Mujahid, M. Impact on the Usage of Wireless Sensor Networks in Healthcare Sector. Int. J. Comput. Sci. Netw. Secur. 2017, 17, 102-105.

20. Atmini, D.; Dwi, L.; Eminugroho, R. Sensitivity Analysis of Goal Programming Model for Dietary Menu of Diabetes Mellitus Patients. Int. J. Model. Optim. 2017, 7, 7-14. [CrossRef]

21. Raimundo, M.S.; Okamoto, J., Jr. Application of Hurst Exponent (H) and the R/S Analysis in the Classification of FOREX Securities. Int. J. Model. Optim. 2018, 8, 116-124. [CrossRef]

22. Alotaibi, M. Investigating the Role of Social Robot in improving diabetic Children Management and awareness. Int. J. Comput. Sci. Netw. Secur. 2017, 17, 121.

23. Khawaja, A.P.; Cooke Bailey, J.N.; Wareham, N.J.; Scott, R.A.; Simcoe, M.; Igo, R.P.; Song, Y.E.; Wojciechowski, R.; Cheng, C.-Y.; Khaw, P.T.; et al. Genome-wide analyses identify 68 new loci associated with intraocular pressure and improve risk prediction for primary open-angle glaucoma. Nat. Genet. 2018, 50, 778-782. [CrossRef]

24. Dian, S.; Rahmadi, R.; van Laarhoven, A.; Ganiem, A.R.; van Crevel, R. Predicting Mortality of Tuberculous Meningitis. Clin. Infect. Dis. 2018, 67, 1954-1955. [CrossRef] [PubMed]

25. Samy, G.; Gamal, N. A Framework for Social Network-Based Dynamic Modeling and Prediction of Communicable Disease. Int. J. Model. Optim. 2019, 9, 30-33. [CrossRef]

26. Lamwong, J.; Pongsumpun, P. Age Structural Model of Zika Virus. Int. J. Model. Optim. 2018, 8, 17-23. [CrossRef]

27. Verdial, F.; Madtes, D.; Hwang, B.; Mulligan, M.; Odem-Davis, K.; Waworuntu, R.; Wood, D.; Farjah, F. A Prediction Model for Nodal Disease among Patients with Non-Small Cell Lung Cancer. Ann. Thorac. Surg. 2019. [CrossRef] [PubMed]

28. Kasiakogias, A.; Tsioufis, C.; Dimitriadis, K.; Konstantinidis, D.; Koutra, E.; Kyriazopoulos, K.; Kyriazopoulos, I.; Liatakis, I.; Mantzouranis, M.; Philippou, C.; et al. P1540Comparison of the European Society of Hypertension stratification and European Society of Cardiology HeartScore for prediction of coronary artery disease and stroke in essential hypertension. Eur. Heart J. 2018, 39, 1540. [CrossRef]

29. Mustaqeem, A.; Anwar, S.M.; Majid, M.; Khan, A.R. Wrapper method for feature selection to classify cardiac arrhythmia. In Proceedings of the 39th Annual International Conference of the IEEE Engineering in Medicine and Biology Society, Jeju Island, Korea, 11-15 July 2017; pp. 3656-3659.

30. Romanowski, K.; Balshaw, R.F.; Benedetti, A.; Campbell, J.R.; Menzies, D.; Ahmad Khan, F.; Johnston, J.C. Predicting tuberculosis relapse in patients treated with the standard 6-month regimen: An individual patient data meta-analysis. Thorax 2019, 74, 291-297. [CrossRef] [PubMed]

31. Alfian, G.; Syafrudin, M.; Ijaz, M.; Syaekhoni, M.; Fitriyani, N.; Rhee, J. A Personalized Healthcare Monitoring System for Diabetic Patients by Utilizing BLE-Based Sensors and Real-Time Data Processing. Sensors 2018, 18, 2183. [CrossRef] [PubMed]

32. Ijaz, M.; Alfian, G.; Syafrudin, M.; Rhee, J. Hybrid Prediction Model for Type 2 Diabetes and Hypertension Using DBSCAN-Based Outlier Detection, Synthetic Minority Over Sampling Technique (SMOTE), and Random Forest. Appl. Sci. 2018, 8, 1325. [CrossRef] 
33. Kieviet, A. Werkzeuge der digitalen Transformation. In Lean Digital Transformation; Springer: Berlin/Heidelberg, Germany, 2019; pp. 57-159.

34. Witten. Weka-Data Mining with Open Source Machine Learning Software in Java. Weka. 2016. Available online: https://www.cs.waikato.ac.nz/ml/weka/ (accessed on 23 May 2018).

35. Fallah, M.; Niakan Kalhori, S.R. Systematic Review of Data Mining Applications in Patient-Centered Mobile-Based Information Systems. Healthc. Inform. Res. 2017, 23, 262. [CrossRef] [PubMed]

36. Padillo, F.; Luna, J.M.; Ventura, S. A Grammar-Guided Genetic Programing Algorithm for Associative Classification in Big Data. Cognit. Comput. 2019, 1-16. [CrossRef]

37. González, J.; Ortega, J.; Damas, M.; Martín-Smith, P.; Gan, J.Q. A new multi-objective wrapper method for feature selection-Accuracy and stability analysis for BCI. Neurocomputing 2019, 333, 407-418. [CrossRef]

38. Muhammad, M.U.; Asiribo, O.E.; Noman, S.M. Application of Logistic Regression Modeling Using Fractional Polynomials of Grouped Continuous Covariates. Niger. Stat. Soc. 2017, 1, 144-147.

39. Dubey, R.; Makwana, R.R.S. Computer-Assisted Valuation of Descriptive Answers Using Weka with RandomForest Classification. In Proceeding of the Second International Conference on Microelectronics, Computing E Communication Systems (MCCS 2017), 76th ed.; LNEE, Ed.; Springer: Singapore, 2019; pp. 359-366.

40. Rani, R.U.; Kakarla, J. Efficient Classification Technique on Healthcare Data. In Progress in Advanced Computing and Intelligent Engineering, 713rd ed.; AISC, Ed.; Springer: Singapore, 2019; pp. 293-300.

(C) 2019 by the authors. Licensee MDPI, Basel, Switzerland. This article is an open access article distributed under the terms and conditions of the Creative Commons Attribution (CC BY) license (http://creativecommons.org/licenses/by/4.0/). 